\title{
Discriminative Pronunciation Learning for Speech Recognition for Resource Scarce Languages
}

\author{
Hao Yee Chan \\ Carnegie Mellon University \\ 5000 Forbes Avenue \\ Pittsburgh, U.S.A. \\ haoyeec@andrew.cmu.edu
}

\author{
Roni Rosenfeld \\ Carnegie Mellon University \\ 5000 Forbes Avenue \\ Pittsburgh, U.S.A. \\ roni@cs.cmu.edu
}

\begin{abstract}
In this paper, we describe a method to create speech recognition capability for small vocabularies in resource-scarce languages. By resource-scarce languages, we mean languages that have a small or economically disadvantaged user base which are typically ignored by the commercial world. We use a high-quality welltrained speech recognizer as our baseline to remove the dependence on large audio data for an accurate acoustic model. Using cross-language phoneme mapping, the baseline recognizer effectively recognizes words in our target language. We automate the generation of pronunciations and generate a set of initial pronunciations for each word in the vocabulary. Next, we remove potential conflicts in word recognition by discriminative training.
\end{abstract}

\section{INTRODUCTION}

In developed countries today, speech recognition systems are ubiquitous. From basic spoken dialog systems (SDS) to complex Google voice search, speech technology can be found everywhere. However, of all the languages in the world, only languages spoken by economically developed nations are available in such systems. Commercial systems such as the Microsoft Speech Server provide high quality recognition but suffer from this lack of diversity. Open-source options such as the CMU Sphinx system offer an alternative but copious amounts of training data and deep knowledge of speech technology are basic prerequisites for applying them to each new language.

Ironically, speech technology offers far more benefits to the developing world than to a mostly literate developed world $[1,2]$. For some low-literate users, speech-based interaction has been found to be more effective than traditional touch-tone interfaces [3]. However, such interaction must be based on high quality automatic speech recognition (ASR) and a well-designed user interface [4]. The problem lies with the development of such ASR given the constraints of technical knowledge and training data availability. Such constraints are exacerbated by the conditions found in the populations speaking these target languages, where literacy rates are low.

The aim of this study is to develop methods to enable speech recognition for resource-scarce languages and aimed at lowliterate users. Given these constraints, the speech recognizer should be:

Permission to make digital or hard copies of all or part of this work for personal or classroom use is granted without fee provided that copies are not made or distributed for profit or commercial advantage and that copies bear this notice and the full citation on the first page. To copy otherwise, to republish, to post on servers or to redistribute to lists, requires prior specific permission and/or a fee.

DEV '12, March 11-12, Atlanta, GA

Copyright (c) 2012 ACM 978-1-4503-1262-2/12/03... \$10.00
- $\quad$ Language-independent

- Cost-efficient

- $\quad$ Easy to use without a need for technical background

- Accurate

- Relatively fast to run

\section{RELATED WORK}

Our work is based upon previous work, Speech-based Automated Learning of Accent and Articulation Mapping (SALAAM) [3, 5].

The Salaam approach aims to provide small-vocabulary recognition by using an existing recognizer in a resource-rich language and cross-language phoneme mapping. The approach removes the need for linguistic experts to provide transliteration of the target "words types" by fully automating the creation of the cross-language phonemic pronunciations. Word types refer to a word or phrase in the target language, which we would like to have in the vocabulary, and which is taken to be a single token for identification. Via a "divide-and-conquer" technique, the approach reduces the search space exponentially, resulting in an accurate yet fast process for deriving cross-language pronunciations.

In earlier work, the SALAAM approach achieved less than $15 \%$ word error rate for a target-language vocabulary of up to 50 word types.

\section{METHOD DESIGN}

\subsection{Cross-Language Phoneme Mapping}

Cross-language phoneme mapping uses acoustics models in a source language to represent corresponding phonemes in a target language. A word type in the target language is defined by a sequence of phonemes in the source language. This sequence of phonemes is taken to be the effective pronunciation of the target word type. For example, the Mandarin (target language) word for "three" might be cross-language mapped to "S AA N" in the MSS U.S. English (source language) recognizer.

It is immediately clear that target language words with sounds which do not have corresponding phonemes in the source language would not be easily represented phonetically. For example, the French word "Trois" has a voiced uvular fricative, which sounds like a mix between " $K$ " and " $H$ " in English. When encountering such difficulties, the sequence of phonemes that best matches the audio samples will be selected.

\subsection{Previously Reported SALAAM Method}

Fang [5] enumerated all combinations of 1 to 3 phonemes to seed his initial pronunciation. The number of word boundaries is fixed at 10 . This means that we allow the recognizer to assume that each audio sample may consist of up to 10 "words", each such word consisting of up to 3 phonemes. 
$* / * / * / \ldots$ OR $* * / * / * / \ldots$ OR $* * * / * / * / \ldots$

where * denotes any phoneme and / denotes word boundaries.

The speech recognizer will return a set $\mathrm{A}$ of phoneme sequences. The top $\mathrm{N}$ results are then taken to be a set $\mathrm{B}$. For each phoneme sequence in $\mathrm{B}$, the first phoneme phl is taken to be a candidate first phoneme for the final result. A new set of phonemes sequences is built for the next iteration by appending ph1 to the combinations above.

ph1 $1 / * / \ldots$ OR ph1 $* * / * / \ldots$ OR ph1 $* * * / * / \ldots$

On this second iteration, the top $\mathrm{K}$ results from $\mathrm{ph} 1 * / * / \ldots$, top $\mathrm{K}$ results from $\mathrm{ph} 1 * * / * / \ldots$ and top $\mathrm{K}$ results from $\mathrm{ph} 1 * * * / * / * / \ldots$ are then combined. The first two phonemes are then appended to the combinations and the cycle repeats. Fang refers to this as the "divide-and-conquer" method.

We terminate the iterations when the best phoneme sequence returned remains the same for three consecutive iterations. This implies that the speech recognizer did not detect any 3 additional phonemes in the speech sample. This final phoneme sequence is then taken to be the result.

\subsection{Current SALAAM Method}

\subsubsection{The Improved SALAAM Method}

Our initial seeding of phoneme sequence is similar to the previously reported method. However, we take all results for the next iteration instead of only the top $\mathrm{K}$. This is because we are concerned that we might prematurely discard potential good phonemes sequences. For example, the $\mathrm{K}+\mathrm{i}^{\text {th }}$ result from ph $1 * * / * / \ldots$ might be better than all the results from ph $1 * / * / \ldots$ If we discard that result immediately, we might have already removed the best phoneme sequence from consideration.

In the second iteration, we build the phoneme sequences as follow:

$$
\text { ph1 } 1 / * / \ldots \text { OR ph1 } * * / * / \ldots
$$

We removed the last phoneme sequence of 3 phonemes in order to compensate for the additional time taken by considering all results instead of just the top $\mathrm{K}$, reducing the time taken by at least a factor of three. Subsequent iterations are similar and the termination criterion remains unchanged.

\subsubsection{Discriminative Training}

Previously, the SALAAM method attempted to find the best phoneme sequence for each word type. These sequences are independent from each other. Given a word, SALAAM will always produce the same phoneme sequence regardless of the other words in the target vocabulary.

We improve the SALAAM method by starting with a list of possible phoneme sequence per word. Our goal is to select a subset of phoneme sequences to minimize any conflict between word types with similar pronunciations. Our motivation is that while a given phoneme sequence may be the best match for a given word type, that pronunciation might be recognized for a different, acoustically similar word type, resulting in unnecessary errors. If we had a less "correct" phoneme sequence which was still recognized for the given word type but avoided being recognized for any other word type in the vocabulary, the word error rate would decrease.

To support such discriminative training, we categorize speech recognition errors into two groups:

Eager errors: an audio sample is recognized by a phoneme sequence which is not the correct word type. This phoneme sequence is then flagged as "eager" for recognition.

Shy errors: a candidate pronunciation (phoneme sequence) is never picked by the recognizer for correct recognition of any of the test samples. This phoneme sequence is then flagged as "shy" or avoids recognition.

We remove all phoneme sequences with eager errors regardless of whether they were correctly recognized. Alternative phoneme sequences would be recognized in turn when these errors were removed.

We also change the recognized phoneme sequence by sorting by confidence score or ranking and returning the top phoneme sequence. We call this word selection.

\subsubsection{Removing Eager Errors}

We present an example in the Urdu language with the word types: ELEVEN, WET, BAD. First we generate a base list of pronunciations for these word types, using the improved SALAAM method (see Table 1).

Table 1. Generated pronunciations for Eleven, Wet and Bad

\begin{tabular}{|c|c|c|c|c|c|}
\hline \multicolumn{2}{|c|}{ Grammar (generated by improved SALAAM) } \\
\hline Word Type & Romanized & \multicolumn{3}{|c|}{ Alternative Pronunciations } \\
\hline \multirow{2}{*}{ Eleven } & Giyaara & J EH T AX & J EH D AX & J EH D AX AX & J EH T AX AX \\
\hline Wet & Bhiga huwa & M D I L AX AX & J U J AA AX & & \\
\hline Bad & Bera & L UH D AX & L UH D AX AX & L UH D AA AX & L U AA AX \\
\hline
\end{tabular}


Table 2. Recognition results based on improved SALAAM

\begin{tabular}{|c|c|c|c|c|c|}
\hline Word Type & Romanized & Sample & \multicolumn{3}{|c|}{ Pronunciation List in Rank Order } \\
\hline \multirow[t]{5}{*}{ Eleven } & Giyaara & 1 & J EH D AA AX & J EH D AX AX & J EH D AX \\
\hline & & 2 & J EH D AX & J EH T AX & J EH T AX AX \\
\hline & & 3 & J EH D AX & J EH T AX & J EH D AX AX \\
\hline & & 4 & J EH T AX & J EH D AX & J EH T AX AX \\
\hline & & 5 & J EH T AX AX & J EH D AX & J EH D AX AX \\
\hline Wet & Bhiga huwa & 1 & J EH D AX & L UH D AX & M D I L AX AX \\
\hline \multirow[t]{5}{*}{$\mathrm{Bad}$} & Bera & 1 & L UH D AX & L UH D AX AX & L UH D AA AX \\
\hline & & 2 & L UH D AX & L UH D AX AX & \\
\hline & & 3 & L UH D AX & L UH D AX AX & L UH D AA AX \\
\hline & & 4 & L UH D AX & L UH D AX AX & L UH D AA AX \\
\hline & & 5 & L UH D AX & L UH D AX AX & L UH D AA AX \\
\hline
\end{tabular}

We use these pronunciations with the baseline recognizer to obtain the following result (see Table 2).

While ELEVEN and BAD are perfectly recognized, the pronunciations for WET have been overshadowed by eager errors (in yellow and orange) from ELEVEN and BAD. We remove these eager pronunciations from the pronunciations and rerun the recognizer. We achieve the desired results (see Table 3).

Our grammar is now of this form (see Table 4).

Table 3. Recognition results after discriminative training

\begin{tabular}{|c|c|c|c|c|c|}
\hline Word Type & Romanized & Sample & \multicolumn{3}{|c|}{ Pronunciation List in Rank Order } \\
\hline \multirow[t]{5}{*}{ Eleven } & Giyaara & 1 & J EH D AA AX & J EH D AX AX & J EH T AX AX \\
\hline & & 2 & J EH T AX & J EH T AX AX & J EH D AX AX \\
\hline & & 3 & J EH T AX & J EH D AX AX & J EH T AX AX \\
\hline & & 4 & J EH T AX & J EH T AX AX & J EH D AX AX \\
\hline & & 5 & J EH T AX AX & J EH D AX AX & J EH T AX \\
\hline Wet & Bhiga huwa & 1 & M D I L AX AX & J EH D AX AX & J U J AA AX \\
\hline \multirow[t]{5}{*}{$\mathrm{Bad}$} & Bera & 1 & L UH D AA AX & L U AA AX & \\
\hline & & 2 & L UH D AA AX & L U AA AX & \\
\hline & & 3 & L UH D AA AX & L U AA AX & \\
\hline & & 4 & L UH D AA AX & L U AA AX & \\
\hline & & 5 & L UH D AA AX & L U AA AX & \\
\hline
\end{tabular}




\subsubsection{Word Selection}

For this example, we reuse the initial grammar (see Table 1). The speech recognizer will return a recognition result for WET (see Table 5). Rank refers to the order of a particular pronunciation in the N-Best list which the recognizer returns. Confidence score refers to the confidence score that the recognizer associates with each pronunciation. We note that the recognizer does not order the returned result by confidence score. This may indicate that other heuristics are used internally by the recognizer to determine the final result. Hence, we decide on the recognizer's final output via 3 possible heuristics.

Top Pronunciation: we return the word type in rank 1, which is ELEVEN.

Occurrences: we return the word type which has the most number of occurrences, which is WET.

Confidence Score: we return the word type which has the highest confidence score, which is BAD.

Table 4. Final grammar after discriminative training

\begin{tabular}{|c|c|c|c|c|}
\hline \multicolumn{5}{|c|}{ Grammar (after discriminating training) } \\
\hline $\begin{array}{c}\text { Word } \\
\text { Type }\end{array}$ & Romanized & \multicolumn{3}{|c|}{ Alternative Pronunciations } \\
\hline Eleven & Giyaara & J EH T AX & $\begin{array}{c}\text { J EH D } \\
\text { AX AX }\end{array}$ & $\begin{array}{c}\text { J EH T AX } \\
\text { AX }\end{array}$ \\
\hline Wet & Bhiga huwa & $\begin{array}{c}\text { M D I L AX } \\
\text { AX }\end{array}$ & $\begin{array}{c}\text { J U J AA } \\
\text { AX }\end{array}$ & \\
\hline Bad & Bera & $\begin{array}{c}\text { L UH D AX } \\
\text { AX }\end{array}$ & $\begin{array}{c}\text { L UH D } \\
\text { AA AX }\end{array}$ & $\begin{array}{c}\text { L U AA } \\
\text { AX }\end{array}$ \\
\hline
\end{tabular}

\section{METHODOLOGY AND RESULTS}

\subsection{Data Collection}

A list of 100 word types was created, consisting of numbers, short commands, diseases, time and other common phrases used in the agricultural domain. The words were chosen to reflect the type of words that are likely to be used in developing world applications with low-literate users. We expanded our vocabulary from the 50 word types used previously to 100 word types because the improved SALAAM method resulted in error rates in the low single digit, which made it difficult to assess the statistical significance of further improvements. A bilingual person in both English and the target language selects the most appropriate or commonly used translation of the word types. The translated list is then used to prompt audio recordings.

The recording was done in a quiet location, through a landline or a cell phone, to replicate the various audio transformations done by telecommunication channels. For this purpose, we have created a simple application running on Voxeo Company's Tropo platform (voxeo.com), where native speakers call a specified telephone number, which triggers the application to call them back by executing a web script. The caller is then prompted to read out the word types, one at a time, in order, going through the entire vocabulary multiple times. We chose not to have the caller repeat the same word consecutively because consecutive repetition of identical words may affect their pronunciation.
We used this method to collect 5 audio samples of each of the 100 word types, from 5 native speakers each. The target language we chose was Urdu, which is the national language of Pakistan, and is spoken widely by some 60 million people in South Asia, many of them low-literate.

Table 5. Recognition results for WET

\begin{tabular}{|c|c|c|c|c|}
\hline $\begin{array}{c}\text { Word } \\
\text { Type }\end{array}$ & Romanized & Results & $\begin{array}{c}\text { Confidence } \\
\text { Score }\end{array}$ & Rank \\
\hline Wet & Bhiga huwa & J EH D AX & 0.803 & 1 \\
\hline & & $\begin{array}{c}\text { L UH D } \\
\text { AX }\end{array}$ & 0.949 & 2 \\
\hline & $\begin{array}{c}\text { M D I L } \\
\text { AX AX }\end{array}$ & 0.753 & 3 \\
\hline & $\begin{array}{c}\text { J U J AA } \\
\text { AX }\end{array}$ & 0.508 & 4 \\
\hline
\end{tabular}

\subsection{Results}

\subsubsection{Baseline}

With the improved SALAAM, we established a baseline error rate. Using the "leave one out" evaluation method, we train the improved SALAAM algorithm on all the data from 4 speakers and test on all the data from the remaining speaker. By varying the vocabulary size and the number of alternative pronunciations, we get the following result (see Figure 1). For $\mathrm{N}$ Alternative Pronunciations, we provide $\mathrm{N}$ possible alternatives. In the case of $\mathrm{N}=1$, only the top pronunciation is used. Based on these results, we decide to use the top 10 alternative pronunciations and the full vocabulary of 100 words as the basis for discriminative training.

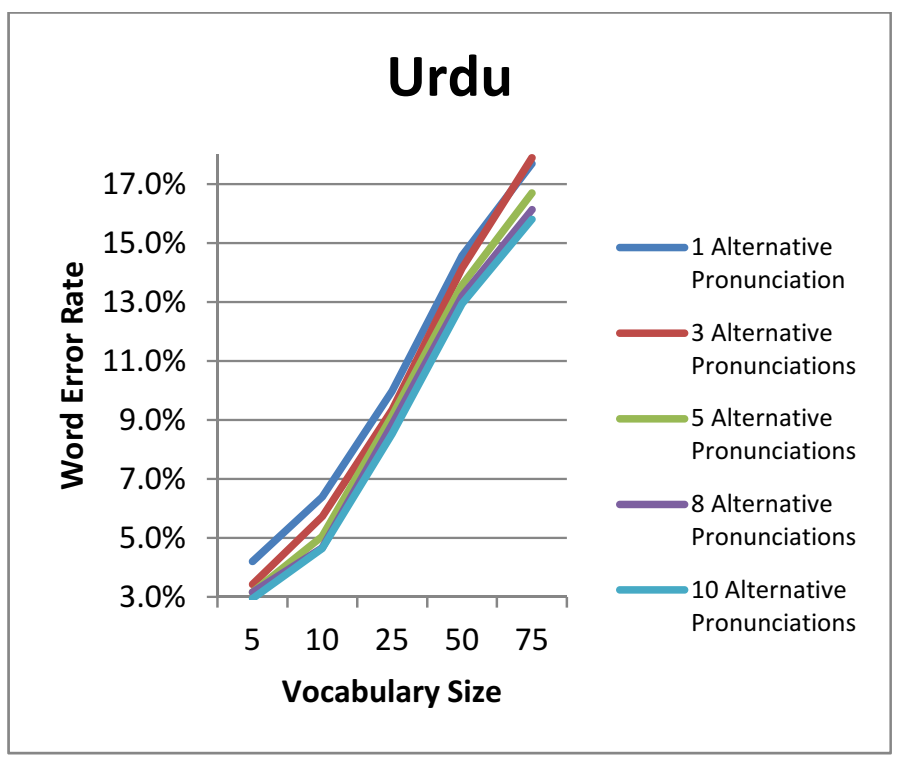

Figure 1. Baseline results on Urdu

\subsubsection{Word Selection}

We tried the three word selection heuristics described in section 3.3.4 on the baseline recognition to derive the following result (see Figure 2). We note that Confidence Score and Number of Occurrences do better than Top Pronunciation. 


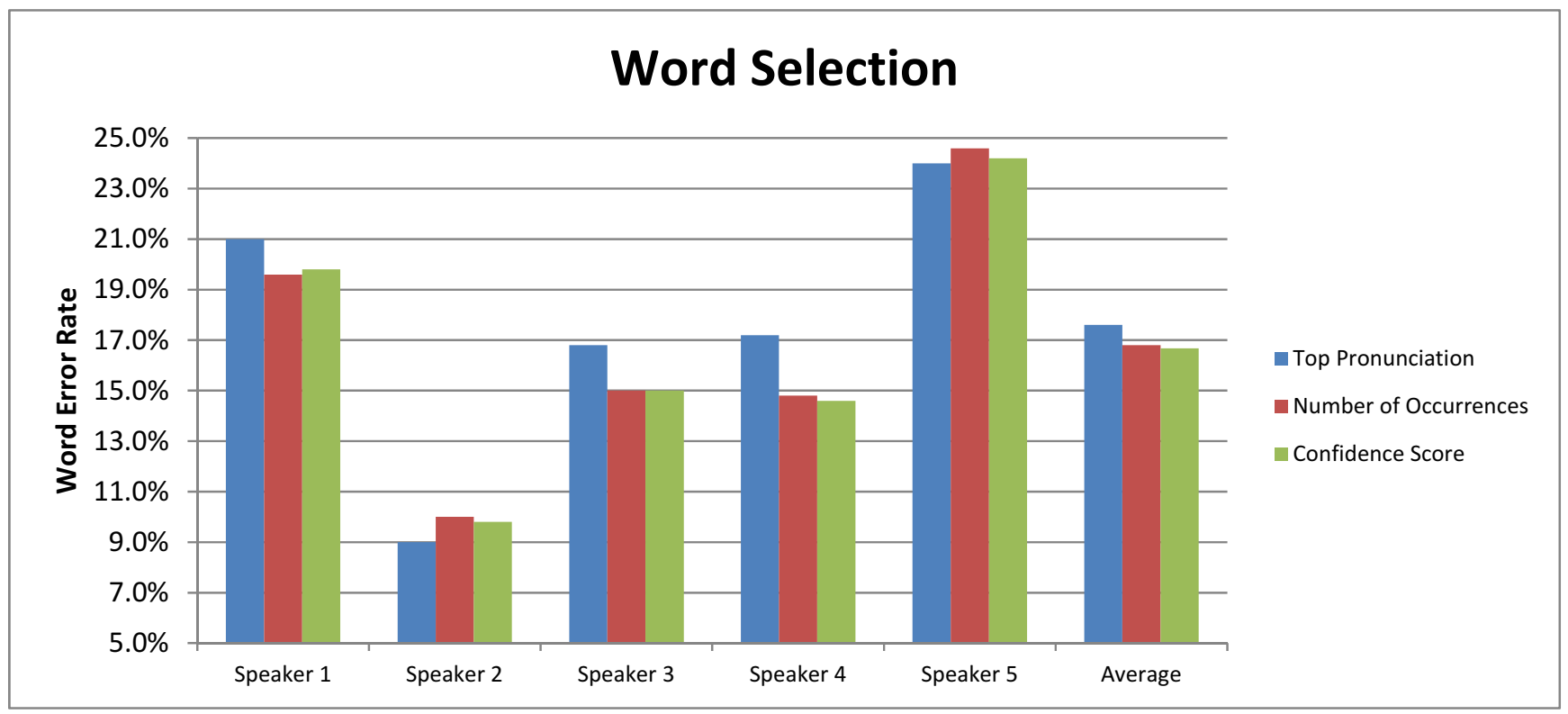

Figure 2. Word selection heuristics on baseline recognition

\subsubsection{Discriminative Training with Word Selection}

Discriminative training was done with two different heuristics, Rank Elimination and Confidence Elimination. Rank Elimination is similar to Top Pronunciation in Word Selection where the top ranked pronunciations are taken to be the recognition result and Confidence Elimination is the counterpart to Confidence Score, where the pronunciations with the highest confidence scores are taken to be the recognition results. Eager errors are then identified from these results and eliminated as described in section 3.3.3. The results using these two discriminative training heuristics in combination with the previously described word selection heuristics are shown in Figure 3.
When combined with discriminative training, the "Top Pronunciation" heuristic did better than the other word selection heuristics despite performing worse on the baseline. From the result, it is clear that discriminative training improves the original SALAAM method significantly. Moreover, the addition of discriminative training does not take a significant amount of time compared to the initial training.

We notice the increase in word error rate after the 8th iteration and believe it to be the result of removing too many pronunciations.

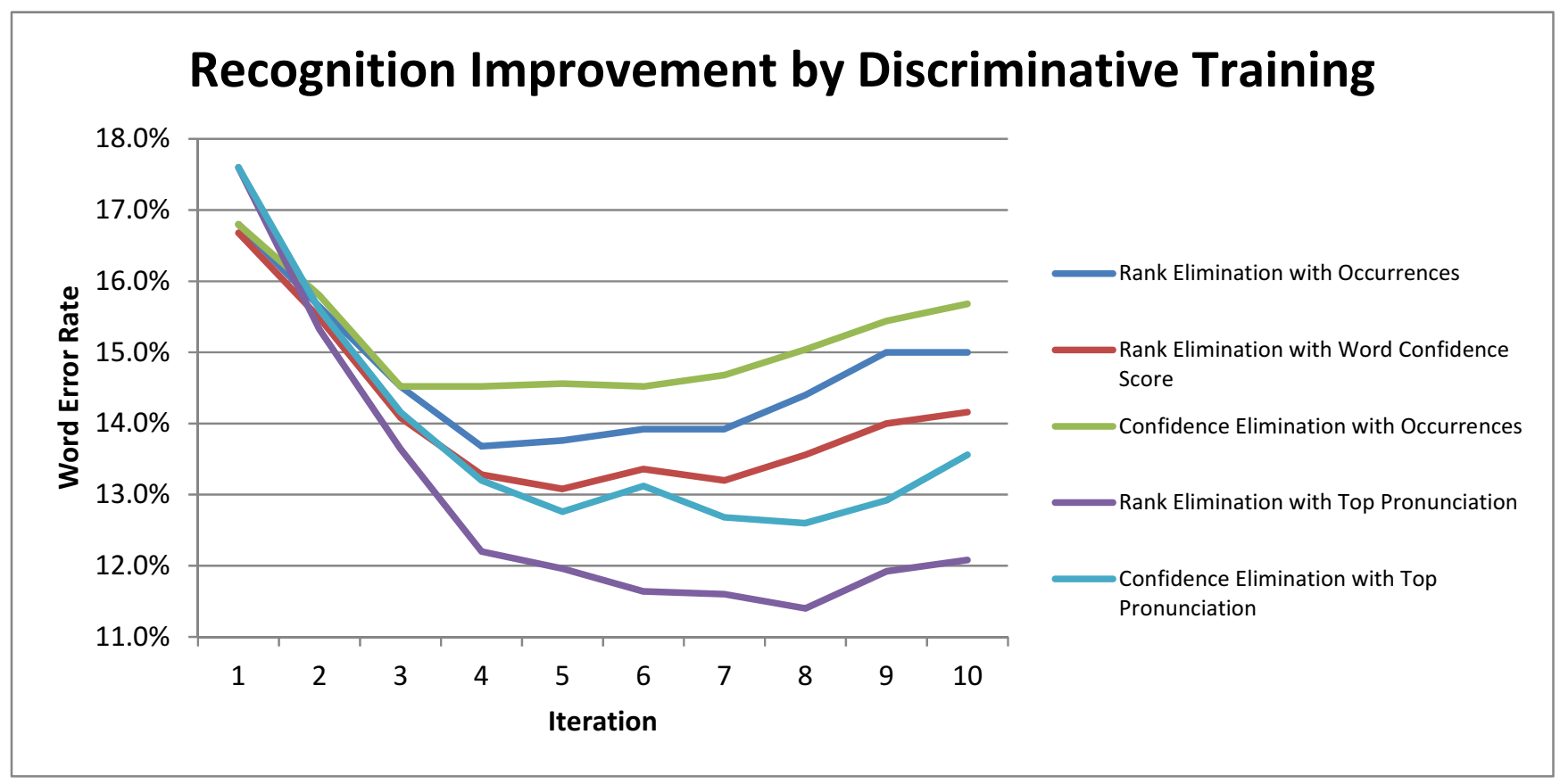

Figure 3. Discriminative training results with various heuristics 


\section{CONCLUSION}

Results show that discriminative training reduces word error rate significantly. While the original SALAAM method performs admirably well, the addition of discriminative training fine-tunes the resulting grammar to reduce word type identification conflicts such as eager errors.

Given that the heuristics are chosen to be independent of source and target languages as well as speech recognizer used, they are sufficiently generic to fulfill our stated objectives.

Although Urdu is the only language tested so far, Urdu falls into our targeted category of a resource scarce language and SALAAM is ready to be deployed for speech recognition applications in Pakistan.

\section{EXTENSIONS}

Additional heuristics and weighting of the rank and confidence score during word selection could improve the results further and should be considered in the future.

It would be useful to develop a web interface to the improved SALAAM, to simplify interaction. Already, audio samples can be uploaded for training purposes as described in section 4.1. This will allow speech recognition technology to reach parts of the world that will truly benefit from it.

\section{REFERENCES}

[1] E. Barnard and M. Davel and G. Huyssteen. 2010. Speech Technology for Information Access: a South African Case Study. Proceedings of AAAI Artificial Intelligence for Development (AI-D’10).
[2] Plauche, M., Nallasamy, U., Pal, J., Wooters, C., \& Ramachandran, D. 2006. Speech Recognition for Illiterate Access to Information and Technology. Proc. 115. International Conference on Information and Communications Technologies and Development, 2006.

[3] Jahanzeb Sherwani, Sooraj Palijo, Sarwat Mirza, Tanveer Ahmed, Nosheen Ali, and Roni Rosenfeld. 2009. Speech vs. touch-tone: telephony interfaces for information access by low literate users. Proceedings of the 3rd international conference on Information and communication technologies and development (ICTD'09), IEEE Press, Piscataway, NJ, USA, 447-457.

[4] Neil Patel, Sheetal Agarmal, Nitendra Rajput, Amit Nanavati, Paresh Dave and Tapan Parikh. 2009. A Comparative Study of Speech and Dialed Input Voice Interfaces in Rural India. Proceedings from CHI 2009 Conference, Boston.

[5] Fang Qiao, Jahanzeb Sherwani, and Roni Rosenfeld. 2010. Small-vocabulary speech recognition for resource-scarce languages. Proceedings of the First ACM Symposium on Computing for Development (ACM DEV '10), ACM, New York, NY, USA, , Article 3, 8 pages. 